\title{
Neptune and Triton: A Flagship for Everyone
}

\section{Lead Author:}

Dr. Abigail Rymer

abigail.rymer@jhuapl.edu, (240) 228-2736

Johns Hopkins Applied Physics Lab

\section{Co-Authors:}

\begin{tabular}{|l|l|l|l|}
\hline \multicolumn{1}{|c|}{ Name } & Home Institution & \multicolumn{1}{c|}{ Name } & Home Institution \\
\hline Kirby Runyon & APL & H. Todd Smith & APL \\
\hline Janet Vertisi & Princeton & Hannah Wakeford & U. of Bristol, UK \\
\hline Kenneth Hansen & NASA HQ & Imke de Pater & Berkeley \\
\hline Krista Soderlund & U. of Texas & James Roberts & APL \\
\hline Kunio Sayanagi & Hampton U. & Jorge Nunez & APL \\
\hline Alan Stern & SwRI & Juan Arrieta & NablaZero lab \\
\hline Lynne Quick & GSFC & Kathleen Mandt & APL \\
\hline Tracy Becker & SwRI & Kevin Stevenson & APL \\
\hline Matthew Hedman & U. of Idaho & Leigh Fletcher & U. of Leicester \\
\hline lan Cohen & APL & Linda Spilker & JPL \\
\hline Frank Crary & U. of Colorado & Mark Hofstadter & JPL \\
\hline Jonathan Fortney & UCSC & Noam Izenberg & APL \\
\hline Adam Masters & Imperial College & Parisi Marzia & JPL \\
\hline Alex Patthoff & PSI & Paul Schenk & LPI \\
\hline Amy Simon & NASA-GSFC & Ralph McNutt & APL \\
\hline Andrew Annex & JHU & Romina Nikoukar & APL \\
\hline Candy Hansen & PSI & Ron Vervack & APL \\
\hline Carol Paty & U. of Oregon & Sarah Moran & JHU \\
\hline Corey Cochrane & JPL & Tom Spilker & n/a \\
\hline Dana Hurley & APL & Tom Stallard & U. of Leicester \\
\hline Elena Provornikova & APL & Abby Azari & Berkeley \\
\hline Elizabeth (Zibi) Turtle & APL & George Hospodarsky & U. of lowa \\
\hline Emily Martin & Smithsonian & & \\
\hline
\end{tabular}

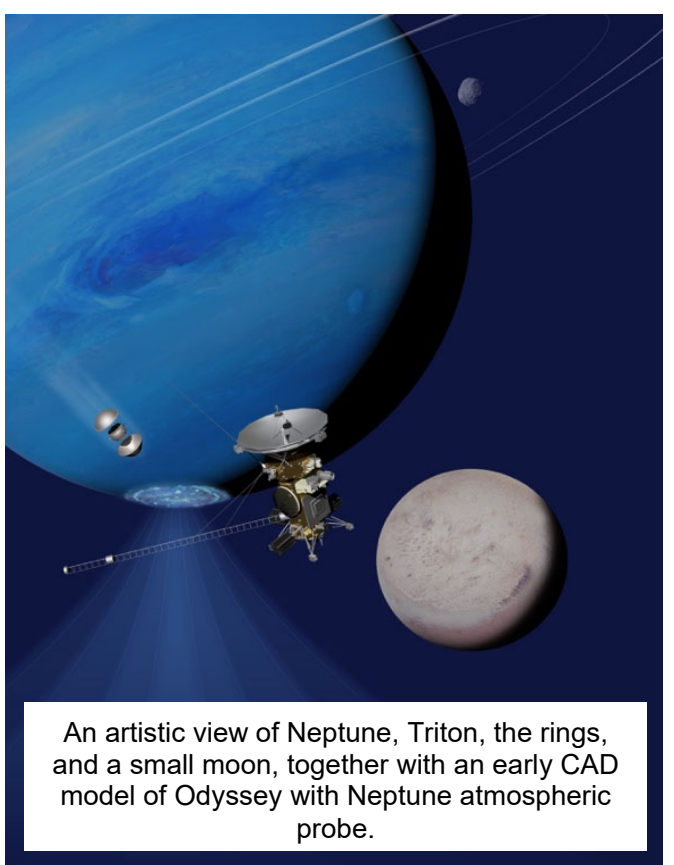

Co-Signers:

\begin{tabular}{|l|l|l|l|}
\hline \multicolumn{1}{|c|}{ Name } & \multicolumn{1}{c|}{ Home Institution } & \multicolumn{1}{c|}{ Home Institution } \\
\hline Heidi B. Hammel & AURA & Emma Woodfield & British Antarctic Survey \\
\hline Sierra Ferguson & Arizona State University & Angela Olsen & University of Oregon \\
\hline A. James Friedson & JPL, Caltech & C T Russell & University of California, UCLA \\
\hline Gordon Bjoraker & & Darrell F Strobel & Johns Hopkins University \\
\hline Bonnie J. Buratti & JPL, Caltech & Amanda R. Hendrix & Planetary Science Institute \\
\hline John F. Cooper & NASA GSFC & Alexander Bader & Lancaster University, UK \\
\hline Dale Cruikshank & & Mark Perry & Johns Hopkins APL \\
\hline Julianne I. Moses & Space Science Institute & Chuanfei Dong & Princeton University \\
\hline Jack Lissauer & NASA Ames Research Center & Cesare Grava & SwRI \\
\hline William B. Hubbard & University of Arizona & Melissa McGrath & SETI Institute \\
\hline Mitchell K. Gordon & SETI Institute & Elias Roussos & $\begin{array}{l}\text { Max Planck Insitute for Solar System } \\
\text { Research }\end{array}$ \\
\hline Matthew S. Tiscareno & SETI Institute & Robert Pappalardo & JPL, Caltech \\
\hline
\end{tabular}


Neptune And Triton: A Flagship for EVERyone - White Paper for Astrobiology and Planetary Science Strategy

\begin{tabular}{|c|c|c|c|}
\hline Name & Home Institution & Name & Home Institution \\
\hline Glenn Orton & JPL, Caltech & Rob Ebert & Southwest Research Institute \\
\hline Timothy E. Dowling & & Ali Sulaiman & University of lowa \\
\hline Olivier Mousis & Aix Marseille Université, France & Laurence Trafton & McDonald Observatory \\
\hline Thomas Greathouse & SwRI & Paul Regensburger & University of Oregon \\
\hline Bryan Butler & National Radio Astronomy Observatory & Karl Mitchell & JPL, Caltech \\
\hline Michele Bannister & U. Canterbury, NZ & Wayne Pryor & Space Environment Technologies \\
\hline Alice Lucchetti & $\begin{array}{l}\text { INAF-OAPD Astronomical Observatory of } \\
\text { Padova }\end{array}$ & Andrew J. Kopf & University of lowa \\
\hline Maurizio Pajola & $\begin{array}{l}\text { INAF-OAPD Astronomical Observatory of } \\
\text { Padova }\end{array}$ & Nick Sergis & Hellenic Space Center \\
\hline Mark Gurwell & $\begin{array}{l}\text { Center for Astrophysics | Harvard \& } \\
\text { Smithsonian }\end{array}$ & Marshall J Styczinski & University of Washington \\
\hline Sebastien Rodriguez & Université de Paris, IPGP & Stuart D. Bale & University of California, Berkeley \\
\hline Davide Grassi & INAF-IAPS, Roma, Italy & Joe Caggiano & University of Oregon \\
\hline Rafael Sfair & UNESP - Sao Paulo State University & Ravindra T Desai & Imperial College London \\
\hline Agustin Sanchez-Lavega & $\begin{array}{l}\text { University of the Basque Country } \\
\text { UPV/EHU, Spain }\end{array}$ & Bertrand Bonfond & Université de Liège \\
\hline David H. Atkinson & JPL, Caltech & Emma Thomas & University of Leicester, UK \\
\hline Shawn M. Brooks & JPL, Caltech & Fritz M. Neubauer & University of Koeln \\
\hline Chloe Beddingfield & NASA Ames / SETI Institute & William Farrell & NASA/Goddard SFC \\
\hline Richard Cartwright & SETI Institute & Brandon Burkholder & Embry-Riddle Aeronautical University \\
\hline Ronald J. Vervack Jr. & Johns Hopkins APL & Georg Fischer & Austrian Academy of Sciences \\
\hline Rob J. Wilson & LASP, University of Boulder Colorado & William Kurth & University of lowa \\
\hline Luke Moore & CSP, Boston University & Philippe Zarka & \\
\hline Joshua Kammer & SwRI & Kevin Baillié & Observatoire de Paris \\
\hline James Sinclair & $\begin{array}{l}\text { Jet Propulsion Laboratory/California } \\
\text { Institute of Technology }\end{array}$ & Mats Holmstrom & Swedish Institute of Space Physics \\
\hline Frederic Allegrini & Southwest Research Institute & Norbert Krupp & $\begin{array}{l}\text { Max Planck Institute for Solar System } \\
\text { Research, Göttingen, Germany }\end{array}$ \\
\hline Krishan Khurana & University of California, UCLA & & \\
\hline
\end{tabular}




\section{A Flagship Mission to the Neptune-Triton System}

Large Strategic Missions, often referred to as Flagship Missions, play a vital role in enabling the pursuit of the most compelling science questions, supporting workforce development, fostering international collaboration, providing opportunities for interdisciplinary investigations, and producing scientific discoveries that inspire the public and the next generations of scientists and engineers [1, 2]. A Flagship Mission to Neptune and Triton covers so many aspects of space exploration that it truly is "A Flagship for Everyone" and we advocate should be the next directed mission supported in the coming decade.

\section{Compelling Science}

A Flagship Mission to Neptune and Triton would provide many firsts, an orbiter and atmospheric probe would not only be feasible on a Flagship budget, but achievable given the current state of the technology required by such a venture [3]. This bold mission of exploration would be the first to orbit an ice giant to study the planet, its rings, small satellites, space environment, and the planetsized moon, Triton, itself a captured dwarf planet from the Kuiper Belt and a geophysically reactivated twin of Pluto. Broadly, the mission would address the following questions: How do the interiors and atmospheres of ice giant (exo)planets form and evolve? (see Section 2). What causes Neptune's strange magnetic field, and how do its magnetosphere and aurora work? (see Section 3 ). What are the origins of and connections between Neptune's rings, arcs, and small moons? (see Section 4). Is Triton an ocean world? What causes its plumes? What is the nature of its atmosphere? and how can Triton's geophysics and composition expand our knowledge of dwarf planets like Pluto? (see Section 5)

\section{Cross-Disciplinary Science Opportunities: Exoplanets and Cruise Phase Science}

We'll start with the science that this mission could do in addition to the goals of the prime mission.

1a. Neptune as an exoplanet. The last several years of exoplanet discovery have taught us that Neptune-sized planets are very common in our galaxy; it is therefore desirable to better understand this class of planets. Importantly, the formation of ice giants remains an open question in the fields of planetary science and astrophysics (e.g. [4] ).

1b. Solar System planets; exoplanets in our backyard. An imaging suite for a Neptune mission, from UV to thermal IR, would provide the capabilities needed for transformational observations of solar system

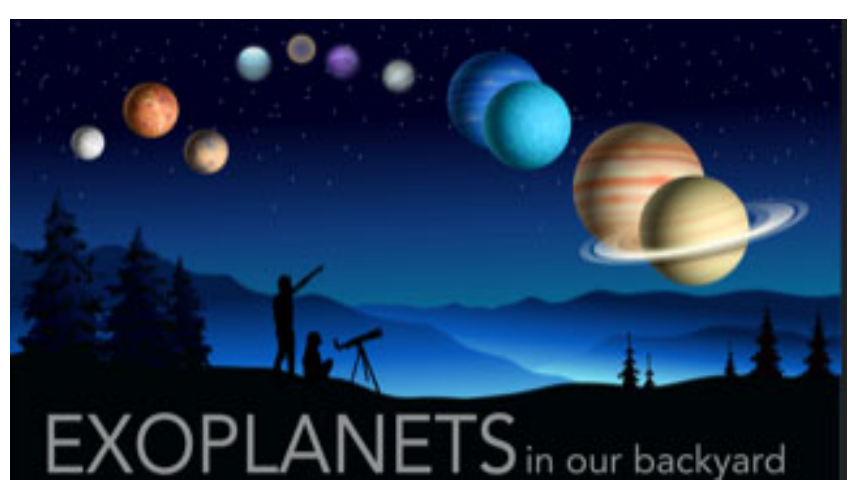

Figure 1 Logo for the cross Assessment Group workshop 'Exoplanets in our Backyard', January 2020 see Arney et al., 2020 planets on the 12- to 16-year cruise phase to Neptune. Disk-averaged unresolved observations are crucial to understanding how our solar system's planets would be viewed "as exoplanets" by a distant observer. See also Harman et al., 2020 and Fortney et al., 2020.

1c. Heliospheric observations. Comprehensive particle and fields instruments, optimized for Neptune, would enable solar wind studies and monitoring of the outer solar system. Data from an 
energetic neutral atom (ENA) camera during cruise and near apoapse during the nominal tour, could provide ENA maps of the heliosphere/interstellar medium (ISM) boundary, e.g. [7]

1d. Centaur/asteroid flyby. A comprehensive remote-sensing suite would also be ideal for making a flyby of a Centaur en route to Neptune. Distant, opportunistic Centaur observations would allow measurements of light curves and composition maps []. Also, a bonus encounter with a Jovian trojan asteroid supplement the Lucy mission may be possible, depending on the launch.

\section{Neptune}

2a. Neptune Interior. Major revelations of the structure and mechanics of the interiors of Jupiter and Saturn from Juno and the Cassini Grand Finale, respectively, are a stark contrast to our ignorance of ice giant interiors [10-15]. While it has long been assumed that the ice giants are water-dominated, this has been called into question with the potential that these planets may instead be "rock-giants" (e.g., Helled \& Fortney, 2020). This distinction has important implications for the formation and evolution of Neptune, the consequences of which can be tested through in situ measurements of noble gas abundances and isotopic ratios of $\mathrm{H}, \mathrm{C}, \mathrm{N}$, and $\mathrm{O}$ (see also Orton et al., 2020). Accurate measurements of Neptune's gravitational field can further constrain planetary composition and the penetration depth of the zonal winds and would be an ideal study in tandem with ground and space based telescopes, for example de Pater et al., 2020.

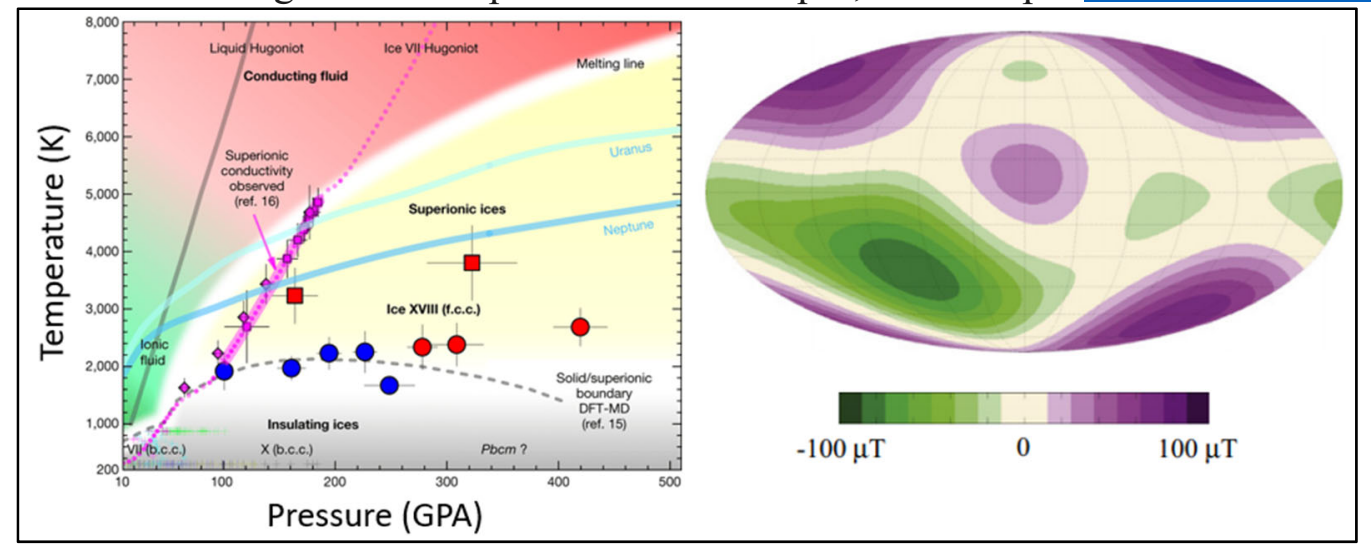

Figure 2. Left: Phase diagram of water showing hypothesized interior conditions for the ice giants [16] magnetic fields are likely generated in the shallow ionic fluid region. Right: Radial magnetic field at the 1 bar pressure level as

measured by Voyager 2, including dipole, quadrupole, and octupole components [17, 18] with purple (green) denoting outward (inward) directed fields.

Voyager 2 also showed that Neptune emits the largest amount of internal heat relative to insolation of all giant planets, especially compared to Uranus' which emits the lowest amount. What is responsible for this inter-ice giant dichotomy and, what regions of the planet are adiabatic? In order to answer these questions, the internal structure must be convolved with thermal evolution for essential determination of the global energy balance and internal heat flow (see also Li et al.g $\underline{2020}$.

A better understanding of planetary composition is directly linked to our understating of its magnetic field. While we know that Neptune's magnetic field is neither dipole-dominated nor axisymmetric, its detailed configuration could not be ascertained by the Voyager flyby and is necessary to determine the novel mechanism of dynamo generation compared to the terrestrial and gas giant planets. See also Soderlund et al., 2020.

2b. Neptune Atmospheric Thermal Structure, Clouds and Hazes. The dominant feature of Neptune's visible-wavelength "photosphere" is its global system of jetstreams that have a westward (i.e., retrograde to planetary rotation) peak at the equator, and an eastward peak in each 
of the north/south hemispheres. This structure is similar to Uranus, and in a stark contrast to Jupiter and Saturn. This dichotomy in the giant planet atmospheric dynamics remains one of the biggest fundamental questions to be answered in geophysical fluid dynamics.

The dynamics and chemistry of a planetary atmosphere are inextricably linked with the thermal structure, which controls which species condense and where, the efficiency of chemical reactions, the nature of instabilities and convective processes, and the 3D shear on atmospheric flows. Each of these processes also feedback to shape the temperature structure, but are currently poorly constrained for Neptune. See also Dahl et al., 2020.

\section{Potential Science Questions - Neptune}

How do giant planets form and evolve?

What is Neptune's internal structure and what regions are adiabatic?

Why does Neptune have a multipolar, non-axisymmetric magnetic field?

Why is Neptune's ratio of emitted/received energy larger than for any other planet

What is the thermal structure and composition and 3D circulation of Neptune's atmosphere?

Suggested Measurement Objectives

- Measure noble gases and isotopic ratios

- Define magnetic and gravity field models, and assess temporal variability

- Establish the energy balance and distribution of internal heat flux

- Create temperature maps as a function of depth

- Map distribution of: tropospheric volatiles, stratospheric hydrocarbons and oxygen species

- Map clouds and haze

- High resolution measurements of wind field

\section{Auroral and Magnetospheric Connections}

3a. Neptune's Magnetospheric Structure and Dynamics. Neptune's magnetosphere is complex, with significant non-dipolar contributions, tilt, and offset from the planet's center. These peculiarities, combined with Neptune's relatively rapid rotational period, lead to widely varying configurations on diurnal and seasonal timescales. In particular, this dynamic behavior tests many precepts in the understanding of planetary magnetospheres relating to magnetic reconnection, energetic coupling to the solar wind, convection and mass transport, and coupling between the magnetosphere and ionosphere. The study of Neptune's aurora and mapping its magnetic field are vital to understanding these processes critical to the Neptune system, including exploring interactions with Triton's neutral cloud, quantifying plasma sources and dynamic processes relevant for supplying and shaping the radiation belts, and directly measuring magnetospheric coupling to Triton to determine if it contains a subsurface ocean.

3b. Thermospheric and Ionospheric Process, and Auroral Drivers and Variability. Our understanding of Neptune's magnetosphere and aurora is limited almost entirely to radio, UV and in-situ observations made by Voyager 2. Neptune produced a complex array of radio emissions, including the most intense, impulsive and narrow-banded emissions ever observed at a radio planet. Voyager 2 also detected extended ultraviolet $\mathrm{H} 2$ band

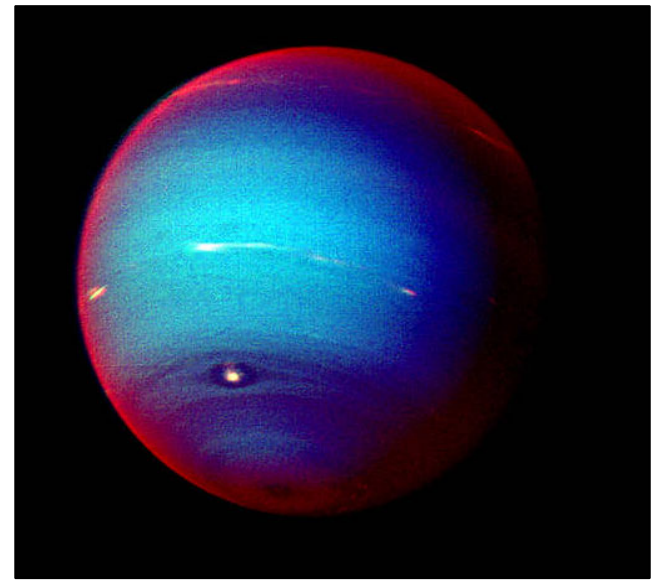

Figure 3. Last image of Neptune taken by Voyager 2 shown in enhanced color. Near the edge of the planet, the haze scatters sunlight at higher altitude, above most of the methane, causing the bright red edge around the planet. 
emissions within Neptune's nightside. This night time airglow was observed across the disk, and was proposed to be powered by photoelectrons flowing along magnetically conjugate field lines that closed in the dayside. Voyager 2 did not include any near-infrared instrument so crucial measurements in that wavelength range ( $\operatorname{such}$ as $\mathrm{H}_{3}{ }^{+}$) remain to be made. See Stallard et al., 2020 and Kollmann et al. 2020 for more details on aurora and magnetospheric measurements.

\section{Science Questions - Aurora \& Magnetosphere Connections}

What dynamic processes drive energy and mass transport in Neptune's magnetosphere?
How are the radiation belts sourced, and what processes determine their structure and energy spectrum?
How do auroral currents flow into and through the ionosphere at Neptune?
Why is Neptune's exosphere so hot?
Suggested Measurement Objectives
- Measure magnetospheric plasma sources and losses and characterize the planetary radiation belts.
- Measure the energy, angular, and compositional distributions of thermal and energetic plasma versus location and
- Meir variability with time.
- - Geariability with time.
- Image the auroral (UV, IR?, Vis?) and ENA emissions.
- Monitor and characterize auroral activity.

\section{Small Satellites and Ring Systems}

Little is understood about the strikingly different ring and satellite systems around Neptune as compared to the relatively well studied Saturn system. Neptune's complex ring dynamics, including the apparent stability some ring arcs harboured within expansive dusty rings, presents particularly intriguing questions about particle interactions and dynamics with direct applications to circumstellar disks. Using ring systems as laboratories for planetary formation processes was a high priority goal for establishing ground truth for exoplanets from Giant Planet studies in the Visions and Voyages decadal survey and we advocate for this to be maintained in the coming decades with particular emphasis on expanding our understanding of ring dynamics by studying the disrupted Neptunian ring system.

The inner regular satellites may have formed with Neptune, such that their composition could indicate available source materials for the planet. The rings, primarily micron-sized dust [27], must be replenished constantly, likely via collisions and/or meteorite impacts on the unobserved "parent" bodies of the rings, as dust has a relatively short lifetime. Ring arcs embedded within the outer Adams ring have been observed to change in brightness, drift in position, or vanish completely [28]. The arcs' stability and confinement are still areas of active

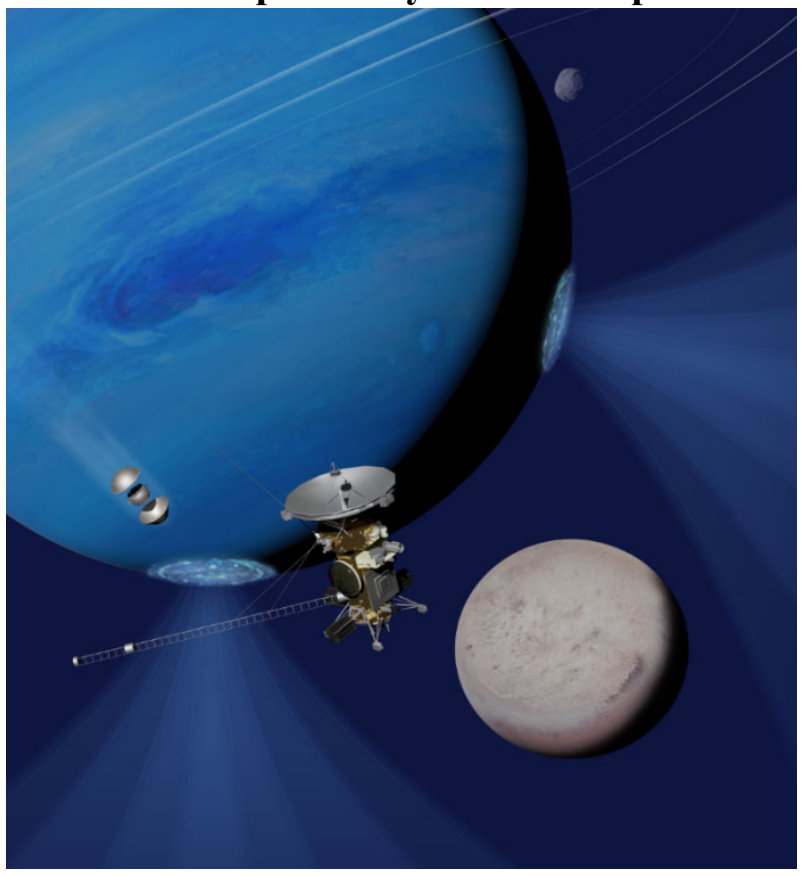

Figure 4. Artist's impression of Neptune, Triton, Rings and peculiar auroral structure, from Rymer et al., 2020. 
research, with solar radiation forces and inelastic particle collisions challenging their maintenance through resonances or co-orbital moonlets [29, 30, 27]. (Note that even Saturn's main rings may be young, possibly not more than $\sim 150$ Myr old [31]). We need to identify the sources of the rings in order to understand their formation through a search for as yet undiscovered moons. The rings can also capture information about Neptune's interior through waves generated in the rings by resonances with the planet's normal modes [Hedman \& Nicholson, 2013].

Some of the irregular moons may be captured objects [32] or remnants of the primordial satellite system perturbed into more complex orbits during the capture of Triton, as is hypothesized for Nereid [33-36]. Compositional analyses would assist in constraining the origins of the satellites. Of particular interest would be the composition and morphology of recently-discovered Hippocamp, which may be a fragment of the larger, near-by moon Proteus [37]. See also Hsu et al., 2020 and Brooks et al., 2020.

\section{Science Questions - Rings \& Satellites}

What is the origin and evolution of the rings and small satellites?

How does the current ring-moon system operate and sustain ring arcs?

Suggested Measurement Objectives

- Search for embedded moonlets

- Identify influences from resonances with satellites \& the planet in the rings

- Observe short-term and long-term variations in the rings and ring arcs

- Determine particle size distribution of the rings

- Constrain photometric properties of the rings and moons

\section{Triton, Dwarf Planet and Candidate Ocean World}

5a. Triton Interior Structure. Does Triton have a subsurface liquid ocean? Recent studies have considered the effects of tidal dissipation in Triton, and have found that both solid body tides and obliquity tides may lead to the preservation of a subsurface ocean until the present day [40-42]. Based on evidence for Triton's young surface $(10-100 \mathrm{MY})$ as observed by the Voyager spacecraft [43], and the possibility of ocean-derived activity, similar to that observed by Cassini at Enceladus, NASA's Roadmap to Ocean Worlds ( priority "candidate ocean world" target, and specifically called out the significance of a mission study to resolve Triton's ocean world status [44].

\section{5b. Triton Surface} Geology and Geophysics. What are the geologic processes responsible for Triton's unique surface features? Although the specific composition of Triton's crustal material is unknown, the surface is punctuated with ridges, cliffs and knobs that extend as high as $1 \mathrm{~km}$ above

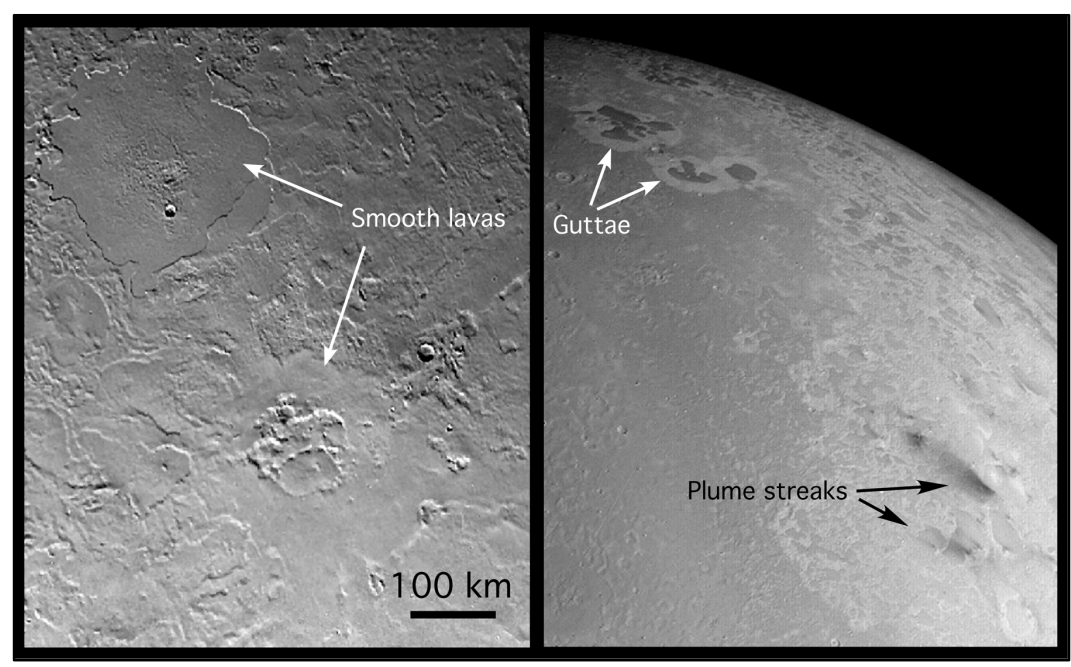

Figure 5. Triton's candidate cryovolcanic features include smooth lavas (left), and guttae and plume streaks (right). Images courtesy of NASA/JPL 
the surface, and by numerous unrelaxed, $\sim 1.5-\mathrm{km}$ deep impact craters. The extent of the surface topography suggests that the crust is composed of rigid materials such as water ice, ammoniawater ice, $\mathrm{CO}_{2}$, and/or $\mathrm{SO}_{2}$ [48-51], all of which are strong enough to preserve high-standing topography at Triton's surface temperatures.

5c. Triton's Atmosphere and Ionosphere. Are Triton's plumes endogenic or solar-driven? Recent data and models motivate a re-examination of the source of Triton's plumes [52]. The Cassini discovery of tidally-driven eruptions confined geographically on Enceladus, and measurements such as vapor mass flux and exit speeds, have expanded possible scenarios for Triton. The possibility that Triton's plumes could be endogenic and sourced from subsurface liquid is deserving of further investigation and would solidify Triton's identity as an ocean world. How does Triton's atmosphere respond to seasonal redistribution of volatiles? What is the nature of Triton's global circulation and climatic response? How does the highly conducting Triton ionosphere interact with the corotating magnetosphere of Neptune? How is Triton's extremely strong ionosphere generated and maintained, and are magnetospheric interactions key?

5d. Triton as a KBO Analog. It has long been recognized [53-55] that Triton, being in a retrograde orbit around Neptune, is almost certainly a captured planet from the Kuiper Belt. Astonishingly, Triton's mass and diameter are slightly larger than any known dwarf planet currently in the Kuiper Belt: $2707 \mathrm{~km}$, vs. $2377 \mathrm{~km}$ for Pluto and $2330 \mathrm{~km}$ for Eris, and only slightly smaller than Europa's $3122 \mathrm{~km}$. See also Hansen et al., 2020.

\section{Science Questions - Triton}

Is there an ocean present, and if so, what is its depth and salinity?

How thick is the ice shell?

What generates the plumes?

What seasonal factors influence Triton's atmosphere and how are they manifest?

How are Triton's surface-atmosphere-magnetosphere coupled?

Suggested Measurement Objectives

- Measure Triton's induced or intrinsic magnetic field and search for temporal variations

- Measure Triton's gravity field

- Measure changes in landforms, plumes, flexure

- Map plumes and plume activity

- Map distribution of Triton surface ices

- Detect and measure scale heights of atmospheric condensed ices, hydrocarbons and N2

- Compute energy input into Triton

\section{Diversity and Inclusion: A Flagship for Everyone}

A balanced portfolio of small (Discovery), medium (New Frontiers) and large class missions is important for NASA [2]. Small and medium sized missions enable the faster cadence and responsivity to new discoveries and have higher risk tolerances. They also require a narrower scientific focus, with one or a few tightly interconnected goals and a limited payload of instruments. Unlike small and medium scale missions, the scale and cost of Flagship missions enable and should require coverage of a broad suite of science objectives, engaging with a large crosssection of the planetary community. For this reason, a mission to Neptune and Triton is ideal for a NASA Flagship. The research described above encompasses (without exaggeration) Planetary Interiors, Atmospheres, Evolution, Magnetospheres, Rings, Small Satellites, Ocean Worlds, Kuiper Belt Objects, Heliophysics, and Exoplanets. The duration of these mission is long, and will 
provide opportunities for engagement with scientists from across these fields in the planetary community, as well as training for the next generation of diversely motivated mission scientists. The science coverage described above for a Neptune-Triton mission is diverse and reflects the growth and evolution of the planetary community.

As described in the science objectives, a Flagship mission to study the Neptune and Triton system provides unique opportunities to interact with scientists from across the planetary community, as well as across disciplines. One strong value-added aspect of the long duration, well instrumented, and directed nature of Flagship missions is the ability to onboard several rounds of new co-investigators via Participating Scientist programs. Participating Scientist programs have been demonstrated to be of critical importance and value to the Planetary Science Division, by increasing intellectual diversity among a project science team and enhancing the science return of the mission [56]. They also provide important career experience, training, and networking opportunities for participants, especially those in the early stages of their career. A perhaps less obvious finding of the above referenced study was that 'Participating Scientist programs also enhance demographic diversity among teams, and are seen as a valuable opportunity for many in the community who may not otherwise have access to mission participation.' Hence it is especially important that Flagship missions engage with a large cross section of research areas, so that Participating Scientist programs can have the greatest impact and that planetary scientists across the community can have the opportunity to benefit from the training, interactions, networking, and career experience. The Neptune-Triton Flagship would provide a diverse suite of science objects, engaging with a broad cross section of the planetary community, and additionally offering the benefits Participating Scientist positions to scientists in a wide range of disciplines.

\section{The Technology is Ready and the Timing is Now}

For complete details of a shovel-ready Neptune-Triton mission, we refer readers to the PMCS report Neptune Odyssey: Mission to the Neptune-Triton System [3, 58] which outlines the timeline for launch, potential payload, cruise and tour, and the overall mission design. We also refer readers to the OPAG White Paper [57] which indicated the high priority for Neptune Flagship mission start in the coming decade, and others describing high level goals [59, $\underline{60}]$.

Following decades of exploration of nearly all the other planets in our solar system we have the instrumentation and expertise, in both modeling and data analysis, necessary to study NeptuneTriton system. We have not revisited an ice giant since our initial passing glimpse with Voyager 2 over 30 years ago; it was mission that inspired curiosity in multiple generations of scientists and planetary enthusiasts alike, and Neptune and Triton beckon us to return and explore. We are ready.

\section{Reference List}

Full reference list can be found here: https://docs.google.com/spreadsheets/d/1ahjqJTKY21DCQAaXheMgrh34IdKuOHXtrq9wwrY4Q $\underline{\mathrm{xl} / \text { edit\#gid=0 }}$ 